\title{
Actualización
}

\section{Consentimiento informado}

\section{Informed Consent}

\section{AUTORA}

\section{PROF. OD. SILVIA INÉS GONZÁLEZ}

Profesora Titular Efectiva Asignatura Clínica Integrada II - Módulo 3 - Clínica Preventiva Interdisciplinaria. Profesora Responsable Cátedra Ética Profesional y Odontología Sanitaria y Legal, Facultad de Odontología Universidad Nacional de Cuyo. Especialista en Docencia Universitaria. Especialista en Odontología Social. Presidenta de la Sociedad Mendocina de Odontología Legal del Circulo Odontológico de Mendoza (COM).

E-mail: sigonzalez00@gmail.com

\section{CO-AUTORAS}

\section{OD. ROSANA RUTH SARLI}

Jefe de Trabajos Prácticos Efectiva Asignatura Clínica Integrada IIMódulo 3 - Clínica Preventiva Interdisciplinaria. Facultad de Odontología Universidad Nacional de Cuyo. Especialista en Docencia Universitaria. Especialista en Odontología Social.

E-mail: rsarli@hotmail.com

\section{OD. PATRICIA VIVIANA RIZZO}

Jefe de Trabajos Prácticos Efectiva Asignatura Clínica Integrada IIMódulo 3 - Clínica Preventiva Interdisciplinaria. Facultad de Odontología Universidad Nacional de Cuyo. Especialista en Docencia Universitaria. Especialista en Odontología Social.

E-mail: pavirizzo@yahoo.com.ar

\section{RESUMEN}

La profesión de odontólogo se ejerce a través de numerosos actos que requieren de la responsabilidad del profesional, relacionado con distintos problemas que ocurren en el quehacer diario en relación a la aprobación por parte del paciente. El consentimiento informado constituye un acto jurídico que debe reunir características propias.

\section{ABSTRACT}

The dental profession is exercised through numerous events that require professional responsibility, related to different problems that happen in daily life in relation to the approval of the patient. Informed consent is a legal act that must meet characteristics.

\section{INTRODUCCIÓN}

La palabra consentimiento, según el Diccionario de la real Academia, significa acción o efecto de consentir. También se entiende por consentimiento el permitir una cosa, conceder que se haga o, asimismo, existencia de conformidad de voluntad entre dos partes. En vez de la palabra consentimiento que viene del latín cum sentire, con sentir, se utilizan para significar los mismo las de licencia, autorización, aprobación o anuen

cia y, en sentido opuesto, como antónimas a dicha palabra las de denegación, disentimiento o prohibición.

Se concibe por consentimiento el acuerdo entre dos voluntades, o sea el consentimiento de una persona o el comportamiento de otra a través de actos que pueden tener consecuencias jurídicas y por lo tanto han de reunir los requisitos objetivos, subjetivos y formales para producir los efectos de dichos actos.

Desde el punto de vista práctico y aplicado a la odontología podemos sintetizar que las actividades profesionales se pueden agrupar en dos clases: 
1). Actos profesionales: que en general no originan daños a los pacientes, como la mayoría de las actividades de la estomatología médica, de la odontología preventiva y otros, para los cuales no hace falta consentimiento expreso por considerarse que al acudir el paciente otorga tácitamente al odontólogo un consentimiento para que realice la historia clínica, las exploraciones y actos terapéuticos que no generen peligro.

2). Intervenciones que implican riesgos: como actos quirúrgicos, extirpación de tumores, exéresis de tejidos, exodoncias de piezas dentales, etc. para las cuales hace falta un consentimiento expreso e información al paciente.

La dificultad estriba en establecer la frontera entre los casos señalados, por lo que en caso de duda, deberá resolverse en el sentido de recabar el consentimiento. (1)

\section{MARCO CONCEPTUAL}

Según la Ley Nacional 26.529 PoderLelislativo Nacional (PLN) "Derechos del Paciente en su Relación con los Profesionales e Instituciones de la Salud". (Sanción: 21/10/2009; Promulgación de Hecho: 19/11/2009; Boletín Oficial 20/11/2009), entiéndase por consentimiento informado, la declaración de voluntad suficiente efectuada por el paciente, o por sus representantes legales en su caso, emitida luego de recibir, por parte del profesional interviniente, información clara, precisa y adecuada con respecto a:

a) Su estado de salud.

b) El procedimiento propuesto, con especificación de los objetivos perseguidos.

c) Los beneficios esperados del procedimiento.

d) Los riesgos, molestias y efectos adversos previsibles.

e) La especificación de los procedimientos alternativos y sus riesgos, beneficios y perjuicios en relación con el procedimiento propuesto. f) Las consecuencias previsibles de la no realización del procedimiento propuesto o de los alternativos especificados.

Art. $6^{\circ}$.- Obligatoriedad. Toda actuación profesional en el ámbito médico-sanitario, sea público o privado, requiere, con carácter general y dentro de los límites que se fijen por vía reglamentaria, el previo consentimiento informado del paciente.

Art. $7^{\circ}$.- Instrumentación. El consentimiento será verbal con las siguientes excepciones, en los que será por escrito y debidamente suscrito:

a) Internación.

b) Intervención quirúrgica.

c) Procedimientos diagnósticos y terapéuticos invasivo.

d) Procedimientos que implican riesgos según lo determine la presente ley.

e) Revocación.

\section{Art. $8^{\circ}$. - Exposición con fines}

académicos. Se requiere el consentimiento del paciente o en su defecto, el de sus representantes legales, y del profesional de la salud interviniente ante exposiciones con fines académicos, con carácter previo a la realización de dicha exposición.

Art. $9^{\circ}$.- Excepciones al consentimiento informado. El profesional de la salud quedará eximido de requerir el consentimiento informado en los siguientes casos:

a) Cuando mediare grave peligro para la salud pública.

b) Cuando mediare una situación de emergencia, con grave peligro para la salud o vida del paciente, y no pudiera dar el consentimiento por sí o a través de sus representantes legales.

Las excepciones establecidas en el presente artículo se acreditarán de conformidad a lo que establezca la reglamentación, las que deberán ser interpretadas con carácter restrictivo.

Art. 10.- Revocabilidad. La decisión del paciente o de su representante legal, en cuanto a consentir o rechazar los tra- tamientos indicados, puede ser revocada. El profesional actuante debe acatar tal decisión, y dejar expresa constancia de ello en la historia clínica, adoptando para el caso todas las formalidades que resulten menester a los fines de acreditar fehacientemente tal manifestación de voluntad, y que la misma fue adoptada en conocimientos de los riesgos previsibles que la misma implica.

En los casos en que el paciente o su representante legal revoquen el rechazo dado a tratamientos indicados, el profesional actuante sólo acatará tal decisión si se mantienen las condiciones de salud del paciente que en su oportunidad aconsejaron dicho tratamiento. La decisión debidamente fundada del profesional actuante se asentará en la historia clínica.

Art. 11.- Directivas anticipadas. Toda persona capaz mayor de edad puede disponer directivas anticipadas sobre su salud, pudiendo consentir o rechazar determinados tratamientos médicos, preventivos o paliativos, y decisiones relativas a su salud.

Las directivas deberán ser aceptadas por el médico a cargo, salvo las que impliquen desarrollar prácticas eutanásicas, las que se tendrán como inexistentes.

\section{DERECHOS DEL PACIENTE}

a) Asistencia. El paciente tiene derecho a ser asistido, sin menoscabo y distinción alguna. El profesional sólo podrá eximirse del deber de asistencia, cuando se hubiere hecho cargo del paciente otro profesional competente.

b) Trato digno y respetuoso. El paciente tiene derecho al respeto de sus convicciones personales, morales y socioculturales y a su intimidad, cualquiera sea su padecimiento.

c) Intimidad. Toda actividad médico asistencial debe observar el respeto por la dignidad humana y la autonomía de la voluntad, así como el debido resguardo de la intimidad del mismo y la confidencialidad de sus datos. 
d) Confidencialidad. El paciente tiene derecho a que toda persona que participe en la manipulación de la documentación clínica, guarde la debida reserva, salvo expresa disposición de la autoridad judicial o por autorización del propio paciente.

e) Autonomía de la Voluntad. El paciente tiene derecho a aceptar o rechazar procedimientos médicos, con o sin expresión de causa, como así también a revocar posteriormente su manifestación de la voluntad. Los niños, niñas y adolescentes tienen derecho a intervenir en los términos de la Ley $N^{\circ} 26.061$ a los fines de la toma de decisión sobre terapias.

f) Información Sanitaria. El paciente tiene derecho a recibir la información sanitaria necesaria, vinculada a su salud. g) Interconsulta Médica. El paciente tiene derecho a recibir la información sanitaria por escrito, a fin de obtener una segunda opinión sobre el diagnóstico, pronóstico o tratamiento relacionados con su estado de salud.

\section{INFORMACION SANITARIA}

La información sanitaria es aquella que, de manera clara, suficiente y adecuada a la capacidad de comprensión del paciente, informe sobre su estado de salud, los estudios y tratamientos que fueren necesarios realizarle y la previsible evolución, riesgos, complicaciones o secuelas de los mismos.

a) Autorización. La información sanitaria sólo podrá ser brindada a terceras personas, con autorización del paciente. En el supuesto de incapacidad del paciente o imposibilidad de comprender la información a causa de su estado físico o psíquico, la misma será brindada a su representante legal, al cónyuge que conviva con el paciente, o la persona que esté a cargo de la asistencia o cuidado del mismo y a los familiares hasta el cuarto grado de consanguinidad. (2)

\section{VALIDEZ LEGAL}

Para que el consentimiento informado tenga validez legal se requiere:

1. Capacidad de consentir (ser mayor de edad y estar psíquicamente apto). Si bien en nuestro país se considera legalmente mayor de edad a una persona con 18 años, G. Berro considera que no puede imponerse a ningún niño o adolescente un procedimiento médico sin existir primero un proceso de información adecuado, permitiéndole expresarse libremente y que su opinión sea tenida en cuenta. El titular es únicamente el paciente y no los familiares, aunque existen excepciones, con esto se busca preservar la confidencialidad del paciente. Con respecto a la capacidad psíquica hay que considerar un aspecto técnico vinculado con los aspectos limitativos de la psiquis del paciente y otro ético, donde se valora la capacidad de entender y valorar la información.

\section{Libre y voluntariamente expresa-} do.

\section{Correcta y suficiente información} acerca del acto médico a realizar por parte del paciente o su representante legal. Si el paciente desconoce los riesgos y las complicaciones de una intervención, no puede asumir la responsabilidad del mismo recayendo en el médico por no haber informado lo suficiente.

4. Concomitante o anterior al acto médico, siendo temporal y revocable sin sujeción a formalidad alguna en los procedimientos de urgencia donde es escaso el tiempo que se dispone, así como el estado psíquico alterado del paciente. Ambas no constituyen las mejores condiciones para una reflexión serena que permita una adecuada elaboración de la información suministrada.

5. Expreso o concluyente, descartándose así el consentimiento presunto. (3)

\section{TIPOS DE CONSENTIMIENTO INFORMADO}

El consentimiento informado puede ser escrito, verbal o implícito. Aunque, es un proceso que requiere una discusión verbal independientemente de si hay o no una forma escrita involucrada.

El consentimiento escrito es para determinados tratamientos invasivos o de alto riesgo, tanto quirúrgicos como de reconstrucciones. Algunos países como también la mayor parte de las aseguradoras de riesgos de trabajo requieren que los formularios sean por escrito. En general se entiende que la persona que otorga el consentimiento es un paciente con mayoría de edad, mientras que los pacientes menores de edad o los adultos con capacidad mental disminuida deben tener consentimiento del tratamiento obtenido de un padre o tutor legal. El adulto que acompaña al paciente pediátrico puede no ser un tutor legal permitido por la ley para dar su consentimiento para los procedimientos médicos. Ejemplos de esto incluyen un abuelo, el padrastro, la niñera o un amigo de la familia. Un niño en un hogar de guarda o tutela del estado puede ser acompañado de un cuidador que pueden o no estar autorizado a dar consentimiento para los procedimientos médicos, de acuerdo con la ley estatal individual. Es recomendable que el profesional de salud obtenga una copia de la orden del juez que nombra al tutor para verificar que esté autorizado para dar su consentimiento al tratamiento médico propuesto. (4)

El consentimiento oral puede ser satisfactorio para determinados procedimientos, por ejemplo un examen dental. Asimismo, el consentimiento verbal debe quedar documentado como discusión diagnóstica con objetivo, descripción, beneficios, riesgos del tratamiento propuesto, opciones alternativas, pronóstico de la ausencia del mismo y los costos. Quedará asentado en la historia clínica que el paciente demuestra haber comprendido toda la información presentada durante el debate y que se obtuvo su consentimiento, para luego adjuntarlo al plan de tratamiento.

El consentimiento implícito, en este 
caso el paciente da el consentimiento a las acciones del profesional sin mencionarlo. Por ejemplo, un paciente se presenta con un dolor bucal, se examina y se le informa que se necesitan radiografías y el paciente permite que sean tomadas sin ninguna objeción. En este caso, el consentimiento está implícito solo en la aceptación por parte del paciente. El elemento clave es que el paciente es consciente del problema y no hizo ninguna objeción cuando comenzó el tratamiento.

El consentimiento implícito es también aplicable en situaciones de emergencia aquí se presta el servicio sin el consentimiento del paciente, es una situación en la que la atención debe prestarse de inmediato para proteger la vida del paciente. (5)

\section{CONCLUSIONES}

Con el correr del tiempo las Ciencias de la Salud han logrado sustituir un modelo clásico de atención como el paternalismo por un nuevo modelo Ila- mado autonomismo. Recordemos que el primero exigía al enfermo obediencia y confianza en un profesional que debía mantener una fuerte autoridad para cumplir con el principio de beneficencia. Mientras que el segundo está basado en una recíproca relación médico-paciente. Actualmente el paciente reclama por sus derechos y autonomía para decidir, mientras que al profesional de la salud se le demanda competencia en las áreas del saber, competencia técnica y competencia ética.

\section{BIBLIOGRAFÍA}

1. MOYA PUEYO, B. ROLDAN GARRIDO, B. SANCHEZ SANCHEZ,J. (1994) Odontología Legal y Forense Ed. MASSON. Barcelona.

2. LEY NACIONAL 26.529 PODER LEGISLATIVO NACIONAL (PLN). Derechos del Paciente en su Relación con los Profesionales e Instituciones de la Salud. Sanción: 21/10/2009; Promulgación de Hecho: 19/11/2009; Boletin Oficial 20/11/2009.

3. GONZÁLEZ DANIEL, GONZÁLEZ HUGO RODRÍGUEZ ALMADA, GUIDO BERRO, ROVIRA. Consentimiento Informado. Análisis crítico de su aplicación en Servicio Quirúrgico Rev. Méd.
Urug. vol.21 no.4 Montevideo dic. 2005 http://www.scielo.edu.uy/scielo. php? script $=$ sci_arttext\& pid $=$ S0303-32952005000400005

4. PEDIATRIC DENTISTRY.Guideline on Informed Consent.

2012 Reference Manual, Vol. 34 Issue 6, p295-297. 3p http://search. ebscohost.com/login. asp $x$ ? direct $=$ true $\mho d b=d d h \& A N=82575032 \nLeftarrow s i$ te $=$ ehost-live

5. GLASSCOE WATTERSON, DIANNE. INFORMED CONSENT AND INFORMED REFUSAL. RDH. Sep2012, Vol. 32 Issue 9, p44-134. 5p. 1 Color Photograph http:/ / search.ebscohost. $\mathrm{com} / \operatorname{login}$ asp $x$ ?direct $=$ true $\& d b=d d h \& A N=80076359 \&$ site $=$ ehost-live 





Página 18

Consentimiento informado

Informed Consent

ABSTRACT

The dental profession is exercised through numerous acts requiring professional responsibility, related to various problems that occur in daily life, considering the patient's approval. Informed consent is a legal act that must have its own characteristics.

Keywords: Dentistry - rights- obligations

Dóning 29 